mals einen Führungsanspruch in Asien erhoben. Wirtschaftlich sind die chinesischen Kommunisten von ihrem Weg überzeugt, und außerdem war Indien eher als China vor dem Konflikt im Úbermut befangen (S. 348). Die Tatsachen zeigen auch, daß die Aufrüstung Indiens schon wegen der ständigen Konfrontation mit Pakistan längst ein notwendiges Úbel war. Indiens Mißerfolg beim Wirtschaftsaufbau trotz vermehrter Entwicklungshilfe aus West und Ost in den letzten Jahren muß noch auf andere politische und soziale Gründe zurückgehen.

Der Fleiß und die akademische Akribie des Buches haben allgemein Beachtung verdient (vgl. z. B. The Times, 8. 10. 70; Observer, 11. 10. 70; Neue Zürcher Zeitung, 20. 11. 70; Der Spiegel, 15. 2. 71; Die Welt, 31. 12. 70). Die indische Empörung über das Buch ist verständlich; doch die Kritik an Maxwell von G. W. (Giselher Wirsing?) in der Suttgarter Zeitschrift „Indo-Asia“ (Heft 1, Feb. 71, S. 95) scheint nicht sachlich. G. W. behauptet vor allen Dingen, daß Maxwell Nehrus Vorschlag über einen Truppenabzug beider Seiten im Westsektor nicht erwähnt und macht ihm ferner den Vorwurf: „Er setzt das unbewohnte Westgebiet der Himalajagrenze mit dem bevölkerten Ostgebiet gleich, ohne zu sehen, daß die Inder in beiden Gebieten wesentlich verschiedene Ziele haben müßten." Tatsächlich hat der Verfasser Nehrus Gegenvorschlag zu Chou En-lais Vorschlag über einen Rückzug der Truppen beider Seiten ausführlich behandelt (S. 138 ff.; vgl. auch S. 430 f.). Da die Chinesen wie gesagt ihre Konzessionsbereitschaft im Ostsektor stets zeigten, scheiterte die friedliche Lösung des Grenzproblems hauptsächlich an dem Beharren Indiens auf seinem Anspruch auf das "unbewohnte Westgebiet". G. W. hat offenbar nicht verstanden, daß Indien eben gleiche Ziele im Ost- und Westsektor hat, diese jedoch mit verschiedener Logik zu begründen versucht, und übersieht die Bedeutung des „unbewohnten Westgebiets" für China wegen der Verbindungsstraße zwischen Sinkiang und Tibet.
Überdies schließt sich G. W. der Ansicht des Direktors des „Indian Institute of Defence Studies and Analyses", K. Subramanyam, an, „daß es Verhandlungen im eigentlichen Sinne (zwischen Indien und China) nie gegeben hat und daß Maxwell den Unterschied zwischen ,Gesprächen' und ,Verhandlungen' nicht kennt". Sowohl Subarhmanyam als auch G. W. haben sicherlich für ihre Rezensionen das Buch von Maxwell nicht Seite für Seite durchgelesen, denn der Verfasser hat die beiden Termini ganz sauber unterschieden, und zwar an wichtigen Stellen sogar mit Anführungszeichen (S. 149, 218, 249 u. a.). Den Nachdruck kann man schon im Index des Buches unter den Schlagwörtern "talks"-not „negotiations" oder „Neh$\mathrm{ru}$, on negotiations/talks" finden.

Y. H. Nieh

\section{RAINER Gerold}

Die Sicherung des Friedens durch die Organisation der Amerikanischen

Staaten (OAS)

Schriften zum Völkerrecht, Heft 14

Duncker und Humblot

Berlin 1971, 204 S., DM 44,60

Es ist zu begrüßen, daß auch im deutschen Sprachraum die lange vernachlässigten Probleme der interamerikanischen Beziehungen langsam mehr Beachtung und Bearbeitung finden (vgl. die erste deutsche Gesamtdarstellung der OAS durch G. Kutzner, 1970, besprochen in dieser Zeitschrift 1971, S. 121). Gerold untersucht einen Teilaspekt aus dem vielgestaltigen Tätigkeitsfeld der OAS, der Beteiligte wie Beobachter stets in besonderer Weise beschäftigt: die Friedenssicherung.

Nach einem kurzen historischen Rückblick packt er das Thema in sinnvoller Systematik unter sechs Gesichtspunkten an: 1. friedliche Beilegung von Streitigkeiten, 2. Selbstverteidigungsrecht, 3. kollektive Sicherheit, Maßnahmen zur Aufrechterhaltung des internationalen Friedens und der Sicherheit, 4. Friedenssicherung bei Bürgerkriegen, 5. Friedenserhaltende Aktionen, 6. Abrüstung, Atomwaffensperrverträge. 
Unter 1. werden die für das interamerikanische System so verworrenen, unübersichtlichen und sich überschneidenden Institutionen, Verfahren und Funktionen, die der friedlichen Streitbeilegung dienen, gut herausgearbeitet, die unwirksamen (Pakt von Bogotá) allerdings ebenso wie die wirksamen. Besonderes Interesse verdient der $\mathrm{Ab}$ schnitt (S. 61 ff.) über das Verhältnis von friedenssichernden Maßnahmen im Rahmen der OAS zu solchen im Rahmen der VN. Obwohl Gerold die politische Funktion des Rekurses an die eine oder andere Organisation für die Gegenwart richtig sieht (S. 72 f.: die lateinamerikanischen Staaten wollen sich von den USA nicht ausschließlich auf die OAS verweisen lassen), hätte der Bedeutungswandel dieses Problems durch die Herausarbeitung der historisch sich jeweils gegenläufig wandelnden Interessen der USA und Lateinamerikas deutlicher gemacht werden können.

Unter 2. erfährt besonders die expansive Interpretation des Selbstverteidigungsrechtes im Rahmen des von den USA forcierten Antikommunismus-Themas angemessene Würdigung und Kritik. Es ist jedoch nicht richtig anzunehmen (S. 96, vgl. aber S. 142 und S. 155), der so (weit) verstandene topos "Selbstverteidigung" habe in der Praxis, z. B. bei den Problemen Kuba und Dominikanische Republik, keine Rolle gespielt.

In dem anderen großen Abschnitt (3.) über die kollektive Sicherheit weist Gerold zwar einleitend (S. 98) nachdrücklich auf den theoretischen Unterschied zur kollektiven Selbstverteidigung hin. Diese Unterscheidung wird aber anschließend nicht recht durchgehalten, was allerdings nicht an mangelnder analytischer Schärfe des Autors, sondern an den interamerikanischen Verträgen wie auch am Begriffe selbst liegt: zwar richtet sich die kollektive Selbstverteidigung grundsätzlich gegen den von außen kommenden Angriff und das Kollektivsicherheitssystem auf die Verhinderung einer Friedensstörung durch die Androhung der kollektiven Aktion al- ler Recht- und Friedliebenden gegen den zum Rechtsbrecher deklarierten Friedensstörer innerhalb des Systems. Es liegt aber auf der Hand, daß die zum Angriff (im Sinne des Selbstverteidigungsrechtes) gesteigerte Friedensstörung auch das echte kollektive Selbstverteidigungsrecht auslösen kann. Die Übergänge sind naturgemäß fließend. Der Autor untersucht sehr sorgfältig an Hand der interamerikanischen Instrumente und der Praxis die damit zusammenhängenden Fragen des Verfahrens, der Beschlußfassung, der möglichen Maßnahmen (Sanktionen) usw. wie auch die Fragen des materiellen Rechts. Hier zeigen sich die erwähnten fließenden Úbergänge in dem Bemühen der OAS (d. h. primär der USA), Art. 6 und ff. Rio-Vertrag, sedes materiae für das kollektive Sicherheitssystem, mit der Antikommunismus-Komponente ideologisch aufzuladen mit der Tendenz, die "Gefährdungen" durch den (internationalen) Kommunismus als "Aggression" hochzustilisieren und die kollektive Sicherheit in kollektive Selbstverteidigung dagegen umschlagen zu lassen. Gerold untersucht die auftauchenden Sachprobleme zunächst im Rahmen des interamerikanischen Systems selbst; anschließend mißt er sie am Maßstabe der Satzung der VN und des allgemeinen Völkerrechts. Die Auseinandersetzungen mit Kuba spielen dabei naturgemäß eine große Rolle. Die gedrängte Erörterung aller relevanten Gesichtspunkte zur sogenannten Quarantäne von 1962 (S. 143 ff.) ist vorzüglich geraten (Castro war allerdings nicht „Präsident", S. 148 und öfter - das war O. Dorticos).

Unter 5. behandelt Gerold, ausgehend von der Praxis der VN, "friedenserhaltende Aktionen" (selbst im Deutschen bekannter als „peace-keeping operations"). Der einzige praktisch gewordene Fall ist der der "Interamerikanischen Friedensstreitmacht" in der Dominikanischen Republik 1965. Auch nach sorgfältiger Analyse kann der Verfasser das Dilemma nicht auflösen, das darin liegt, daß regionale peace-keeping operations zwar „im Prinzip“ nach den interamerikanischen Verträgen zulässig sein mö- 
$\operatorname{gen}^{1}$ (S. 170-174), daß die OAS-Streitmacht aber „eigentlich" nicht den hierfür entwickelten Kriterien des Konsensus usw. entsprach, weil die vorhandenen dominikanischen Autoritäten gegen sie protestierten, weil die USA die OAS präjudiziert hatten und diese nicht mehr frei entscheiden konnte, weil die OAS der Streitmacht - was der Verfasser richtig sieht - vielmehr nur deshalb zustimmte, um wenigstens nachträglich eine Spur von Einfluß und Kontrolle auszuüben. Wenn der Verfasser sie dennoch letztlich für gerechtfertigt hält, weil sie nicht gegen die Dominikanische Republik gerichtet gewesen sei, sondern ihr den Weg der demokratischen Selbstbestimmung habe offenhalten sollen (S. 175 - vgl. die Besprechung des $\mathrm{Bu}$ ches von Moreno in diesem Heft), so macht er sich letztlich die offizielle Argumentation der USA zu eigen, die er selbst an anderer Stelle (S. 168 Anm. 51) $\mathrm{zu}$ Recht „zynisch“ nennt. Insgesamt ist die Arbeit ebenso juristisch exzellent wie einseitig. Für die Aufgabe, in Lateinamerika den Frieden zu sichern, enthalten die geschilderten Instrumente und Verfahren nun einmal nicht die relevantesten Aspekte.

Knud Krakau

\section{Jose A. Moreno}

\section{Barrios in Arms. Revolution in Santo Domingo}

University of Pittsburgh Press 1970

XIV, 226 S., $\$ 8,95$

Das Buch ist in zweifacher Hinsicht wichtig, einmal im Hinblick auf die Strategie der Revolution in Lateinamerika, obwohl Moreno dazu ausdrücklich nichts sagt. Er beschränkt sich völlig auf die Analyse der dominikanischen Ereignisse von 1965. Hier liegt die andere Bedeutung der Arbeit als Fallstudie dieser Ereignisse trotz der schon vorhandenen Bücher von Wiarda, Draper, Niedergang, Kurzman, Szulc, Martin u. a. Moreno behandelt nicht die Aktionen aller Beteiligten (außer der
Dominikanischen Republik der USA, VN, OAS usw.) als internationales Ereignis. Vielmehr berichtet und analysiert er die dominikanischen Geschehnisse gewissermaßen von ihrer inneren Bewegungskraft her, nämlich dem Volk der Dominikaner. Er liefert die (politisch-soziologische) Anatomie der dominikanischen "Revolution“. Der Verfasser ist dafür in besonderer Weise geeignet: geboren in Kuba, erlebte er als ausgebildeter Soziologe, mit Feldarbeit befaßt, die kritischen Monate im Zentrum des Aufstandes in Santo Domingo. Ausgangspunkt der Ereignisse war die Planung eines für Lateinamerika fast traditionellen Staatsstreiches mit der etwas selteneren Variante, daß er sich gegen das usurpatorische militärisch-zivile Regime Reid Cabral richtete und die Wiederherstellung der Verfassung von 1963 sowie die Wiedereinsetzung des gewählten Präsidenten J. Bosch zum Ziele hatte. Planer waren kleine Gruppen von Bosch-Anhängern, hauptsächlich aus seiner Partei PRD, und von jüngeren Offizieren, Mittelstandselite, liberal bis sozialdemokratisch orientiert. Die zufallsbedingte vorzeitige Auslösung der Ereignisse (am 24. April) interessanterweise taucht das revolutionäre Symbol des Bastille-Sturmes wieder auf - schuf Überraschung und allgemeine Konfusion, die aber alle Beteiligten viel spontaner und echter ihre durch Ideologie, Systemstellung und Interesse bedingte Rolle finden und spielen ließ, als dies sonst möglich gewesen wäre. Der weitere Ablauf ist bekannt: die militärischen Stützen des Regimes Reid hatten nichts gegen dessen Ablösung durch einen "konstitutionalistischen " provisorischen Präsidenten, widersetzten sich jedoch der von den Rebellen unabdingbar geforderten Reaktivierung der Verfassung von 1963 und der Rückkehr Boschs, griffen dann die Rebellen während der Verhandlungen an und lösten damit einen Massenaufstand der Bevölkerung aus, der ohne die Intervention der USA am 28. April

1 Vgl. jetzt dazu James R. Jose, An Inter-American Peace Force within the Framework of the Organization of American States: Advantages, Impediments, Implications (Metuchen, N. J. 1970). 